\title{
Co-Modeling and Co-Simulation of Package and On-Chip Decoupling Capacitor for Resonant Free Power/Ground Network Design
}

\author{
Hyunjeong Park, Hyungsoo Kim, Dong Gun Kam, and Joungho Kim \\ Terahertz Interconnection and Package Lab., Dept. of Electrical Engineering and Computer Science \\ Korea Advanced Institute of Science and Technology, 373-1 Guseong-dong, Yuseong-gu, Daejeon 305-701, KOREA \\ E-mail: teralab@ee.kaist.ac.kr, angel820@eeinfo.kaist.ac.kr, Tel: +82-42-869-5458, Fax: +82-42-869-8058
}

\begin{abstract}
The co-modeling and co-simulation results about the package and on-chip power/ground network have been demonstrated. The inevitable parallel resonance peak, due to the inductive parasitic on the package and on-chip decoupling capacitor, was analyzed in frequency domain. Subsequently, the co-design procedure for the resonant free power/ground network was suggested and evaluated simply in frequency domain.
\end{abstract}

\section{Introduction}

In modern CMOS technology, a large number of logic blocks and I/O blocks operate in higher speed and demand more power while the operating voltage is lowered continuously. Therefore, considerable current consumption has been occurred in a system within the short transition time. The parasitic inductance and the parasitic resistance existing on the on/off-chip power/ground distribution network generate significant voltage ripple, so called simultaneous switching noise (SSN), which is proportional to di/dt, and IRdrop, respectively.

As a result, for the effective reduction of SSN and IRdrop, several kinds of decoupling capacitors have been equipped between power and ground distribution networks on a chip, a package, or a PCB. [1]

However, adding decoupling capacitors on a chip gives rise to a parallel resonance at mid-frequency where both the capacitance of the on-chip decoupling capacitor and the parasitic inductance of the power/ground network on a package become the same impedance.

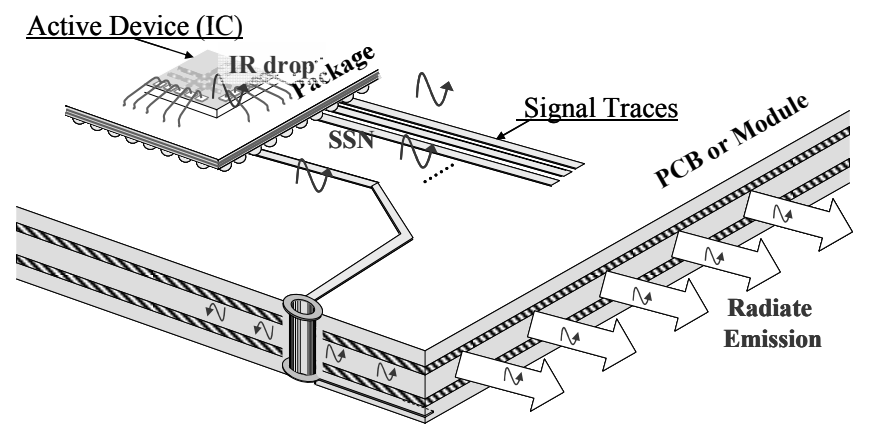

Fig. 1 The parallel resonance can induce the unintentional increase of power/ground noise or unexpected EMI problem.

As shown in Fig. 1, this parallel resonance can induce the unintentional increase of power/ground noise or unexpected EMI problem [2]. In other words, the parallel resonance peak becomes another noise source to high-speed digital systems which will create fatal errors, for instance, logic failure, jitter occurrence, or I/O reference level instability. Therefore, the co-design of a package with on-chip decoupling capacitors is strongly required to remove this resonance peak

In this paper, we analyze the effect of resonance peak first, and subsequently, propose the optimized co-design method for resonant free power/ground network. In order to achieve a more accurate design, we have performed on the comodeling and the co-simulation of a package and on-chip decoupling capacitors. In addition, this work takes every parasitic component into consideration. There have been several previous works to investigate the resonant free power distribution network, such as the adaptive voltage positioning (AVP) [3]. These previous works, however, ignored the onchip parasitic components and focused only on the off-chip design.

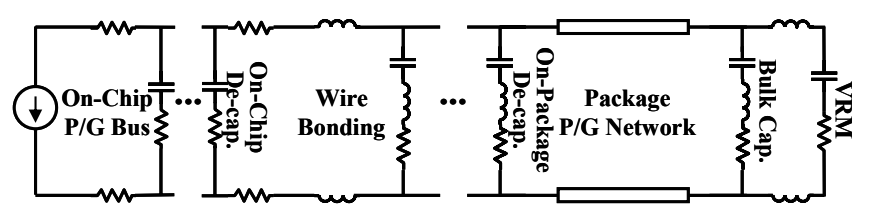

Fig. 2 Simplified circuitry of power/ground network

\section{Co-modeling of the Package and On-chip Power/Ground Network}

The simplified circuitry of power/ground network is depicted in Fig. 2. In this section, each part of power/ground network will be modeled in detail. In order to model the power/ground structures of the package substrate and the metal bus inside the chip, the balanced TLM (Transmission Line Matrix) method is used. The model for the on-chip decoupling capacitor is based on the CMOS technology.

\section{a. Modeling of the Package Power/Ground Plane}

In general, TLM is the high frequency modeling method that the arbitrary plane structure is represented by the combination of the ideal transmission lines. If the length of one transmission line is shorter than the $1 / 10$ of wavelength, the lumped elements can substitute the transmission lines. Using the lumped elements make possible, the intuitive analysis for the circuitry and saves considerable time as well. In the balanced TLM method, the ideal ground in the general TLM model is removed. The resistance and inductance in power/ground plane are divided, and this makes it possible to analyze the multi-layer package and PCB structures. [4]

Fig. 3 shows the balanced TLM model for the package substrate. In Fig. 3(a), the conceptual model for the 
power/ground plane (40um by 40um) is shown. Between the power and the ground plane, it is filled with the dielectric material, which has the dielectric constant, 4.5. The structure for one TLM cell is presented in Fig. 3(b), and each parameter, R, L, C, G, represents the conductor loss, inductive coupling, capacitive coupling, and dielectric loss, respectively. Here, the inductance is $753.98 \mathrm{pH}$ and the capacitance is $1.66 \mathrm{pF}$. Since the resistance and conductance values are frequency dependant, the values are calculated by the equations, Eq. (1a) and Eq. (1b) respectively, as follows.

$$
\begin{aligned}
& \mathrm{R}=2 \sqrt{\frac{\pi \mathrm{f} \mu}{\sigma}}, \\
& \mathrm{G}=2 \pi \mathrm{f} * \mathrm{C} * \tan \delta
\end{aligned}
$$

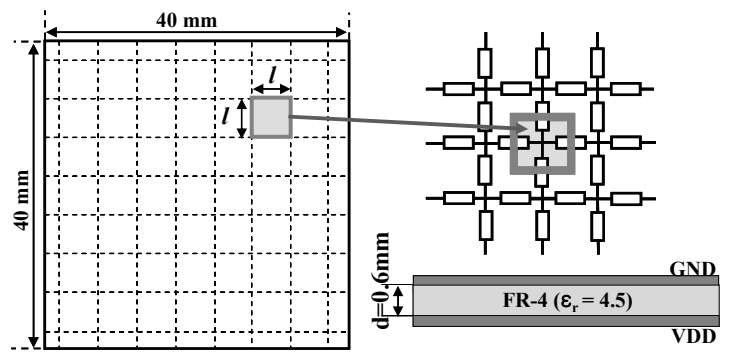

(a)

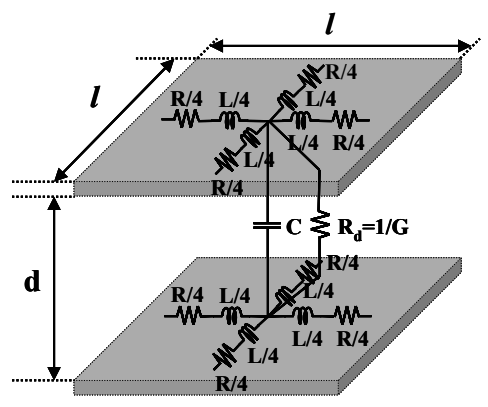

(b)

Fig. 3 The balanced TLM model for the package substrate ; (a) the conceptual model for the power/ground plane, (b) the structure for one TLM cell

\section{b. Modeling of the On-chip Power/Ground Bus}

The model for the on-chip power/ground bus also introduces the balanced TLM method. General on-chip power/ground network has the mesh structure as shown in Fig. 4(a). From the electrical and physical information depending on the conditions such as the sheet resistance, metal thickness, pitch, and stack-up, the parameter values are defined for one cell. The resistance is extracted from the sheet resistance. The capacitance refers to the capacitive coupling in the cross parts between the power bus and the ground bus. Therefore, the resistance and the capacitance is defined by,

$$
\mathrm{R}=\frac{2 \rho}{\mathrm{d}}
$$

$$
\mathrm{C}=\varepsilon_{0} \varepsilon_{r, S i} \frac{\mathrm{s}^{2}}{\mathrm{~d}}
$$

Using this model, the noise distribution can be observed 2-dimensionally when the drivers and the on-chip decoupling capacitors at the arbitrary positions operate. This model ignores the capacitive coupling to substrate. Actually, in this paper, since the analysis is concentrated on the self impedance of the power/ground network, the substrate coupling can be dismissed.

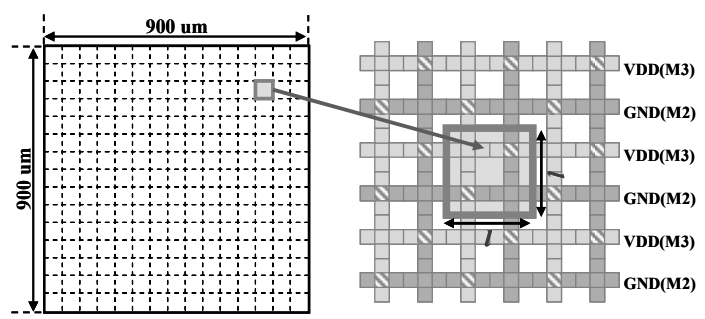

(a)

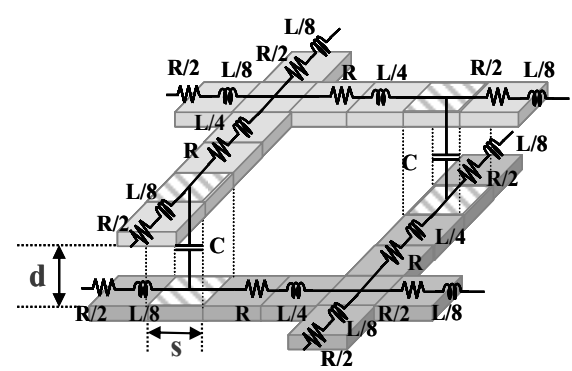

(b)

Fig. 4 The model for the on-chip power/ground bus; (a) the on-chip power/ground network with the mesh structure, (b) the structure for one mesh cell

\section{c. Modeling of the On-chip Decoupling Capacitor}

As expected by Fig. 2, the best way to make the low impedance seen at the chip is to insert on-chip decoupling capacitors with high capacitance. Common CMOS technology uses the MOS capacitor as the on-chip decoupling capacitor which utilizes the capacitance between gate and silicon. However, the parasitic resistance of the on-chip decoupling capacitor cannot be neglected and its accurate modeling is needed.

Fig. 5 shows the physical structure and the equivalent circuit model of the on-chip decoupling capacitor. As shown, the on-chip decoupling capacitor is implemented by the connection between the drain node and the source node.

The on-chip decoupling capacitor is represented by the equivalent circuit like Fig. 5(b) which consists of the capacitance and the ESR (Equivalent Series Resistance). The values are calculated by the following equations, Eq. (3a) and Eq. (3b). [5]

$$
\mathrm{C}_{\mathrm{ox}}=\frac{\varepsilon_{\mathrm{SiO}_{2}} \varepsilon_{0}}{\mathrm{t}_{\mathrm{ox}}} \times \mathrm{WL}
$$




$$
\begin{aligned}
\mathrm{ESR} & =\mathrm{R}_{\mathrm{G}}+\mathrm{R}_{\text {ch }} \\
& =\mathrm{R}_{\text {poly }}\left(\alpha \frac{\mathrm{W}}{\mathrm{L}}+\beta\right)+\frac{1}{\gamma} \cdot \frac{1}{\mu_{\mathrm{n}} \mathrm{C}_{\text {ox }}\left(\mathrm{V}_{\mathrm{G}}-\mathrm{V}_{\mathrm{T}}\right)} \cdot \frac{\mathrm{L}}{\mathrm{W}} \\
& \approx \frac{1}{12}\left(\mathrm{R}_{\text {poly }}\left(\frac{\mathrm{W}}{\mathrm{L}}\right)+\frac{1}{\mu_{\mathrm{n}} \mathrm{C}_{\text {ox }}\left(\mathrm{V}_{\mathrm{G}}-\mathrm{V}_{\mathrm{T}}\right)} \cdot\left(\frac{\mathrm{L}}{\mathrm{W}}\right)\right)
\end{aligned}
$$

where,

$\mathrm{R}_{\text {poly }}$ : gate (ex. poly) sheet resistance

$F_{\mathrm{n}}$ : electron mobility $\left(\sim 300 \mathrm{~cm}^{2} / \mathrm{V} \cdot \mathrm{s}\right)$

$\mathrm{V}_{\mathrm{G}}$ : gate bias voltage

$\mathrm{V}_{\mathrm{T}}:$ threshold voltage

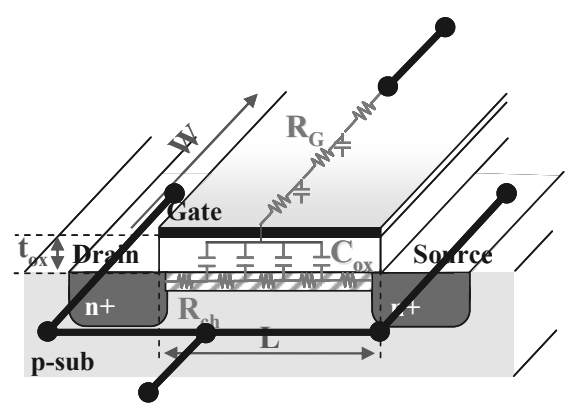

(a)

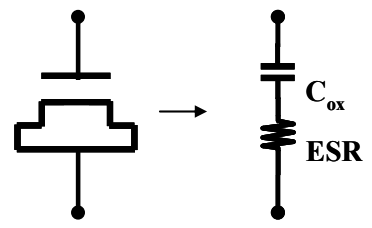

(b)

Fig. 5 (a) The physical structure and (b) the equivalent circuit model of the on-chip decoupling capacitor

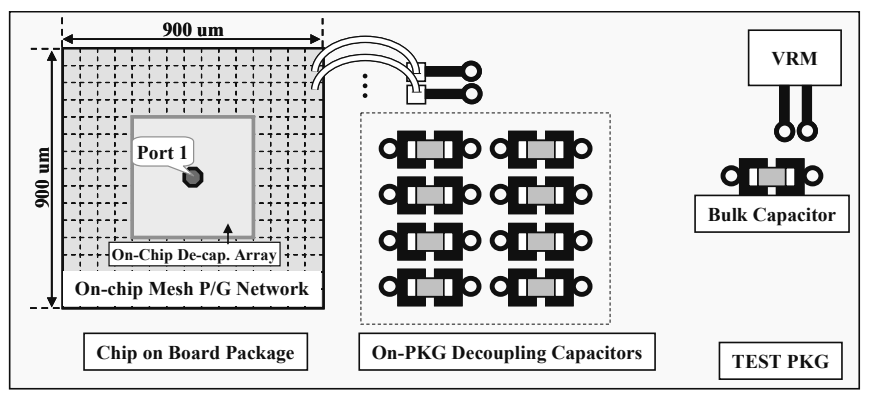

Fig. 6 The setup for analyses of the mid-frequency resonance

\section{Analysis of the Mid-frequency Resonance}

In this section, the analysis of the mid-frequency resonance is described. Using the model for the package and the on-chip, the self impedance of the power/ground network is observed at the position of the on-chip. The tendency of the mid-frequency resonance has been demonstrated with the impedance plots, by varying the size and position of decoupling capacitor, the sheet resistance of the on-chip power/ground bus, the bond-wire inductance, and so on. Fig. 6 shows the setup for the analysis. The noise source with onchip decoupling capacitor array in $1600 \mathrm{~mm}^{2}$ is located at port 1 .

\section{a. On-Chip Decoupling Capacitor Effect}

Initially, the effect of the on-chip decoupling capacitor is demonstrated in Fig. 7. Here, the parallel resonance peaks are observed at around $200 \mathrm{MHz}$. This resonance peak comes from the inductance of the package power/ground network and the capacitance of the on-chip decoupling capacitor. The impedance curves after the resonance frequency become lower as the number of the on-chip decoupling capacitors, in the same chip area, increases.

As described earlier, the ESR (equivalent series resistance) of the one on-chip decoupling capacitor is the summation of gate poly resistance and channel resistance. Since the practical value of the ESR is at least several tens $\mathrm{ohm}$, the total ESR value needs to be reduced by the parallel combination of the decoupling capacitors. However, in the same chip area, as the number of the on-chip decoupling capacitor increases, the more routing area, for additional drain and source connection, has to be demanded. Consequently, the total capacitance is decreased with the increase of the parallel combination in the same area.

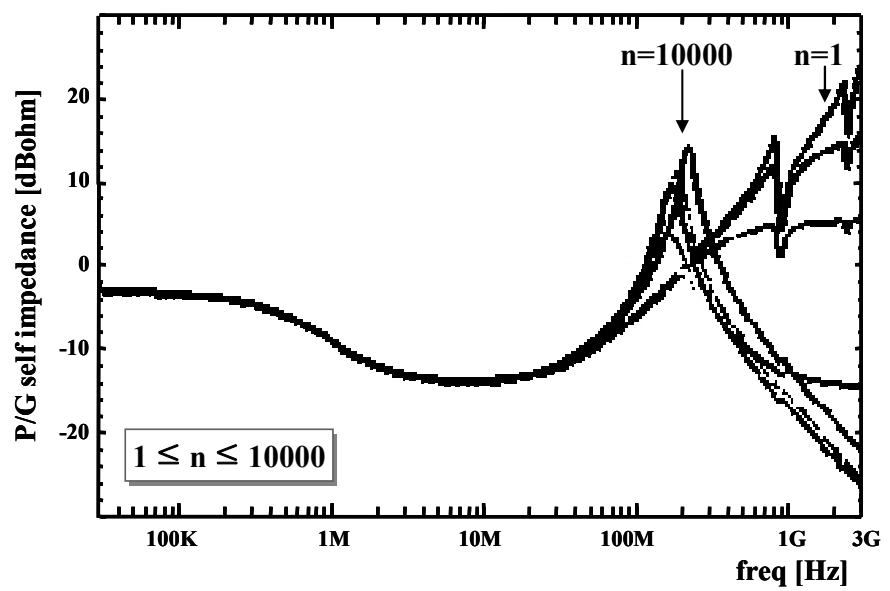

Fig. 7 The effect of on-chip decoupling capacitor parallel combination on power/ground impedance

\section{b. Wire Bonding Effect}

Fig. 8 shows the effect of the number of bond-wires. As the number of the bond-wires increases, the resonance frequency shifts to a higher frequency. The parallel combination of the bond-wires causes the total inductance to be lowered. The impedance at the resonance peak also lowers as the number of the bond-wires increase because the Q-value is proportional to the inductance. However, in most cases, the increase in the number of the bond-wires has limitations due to the cost issues. 


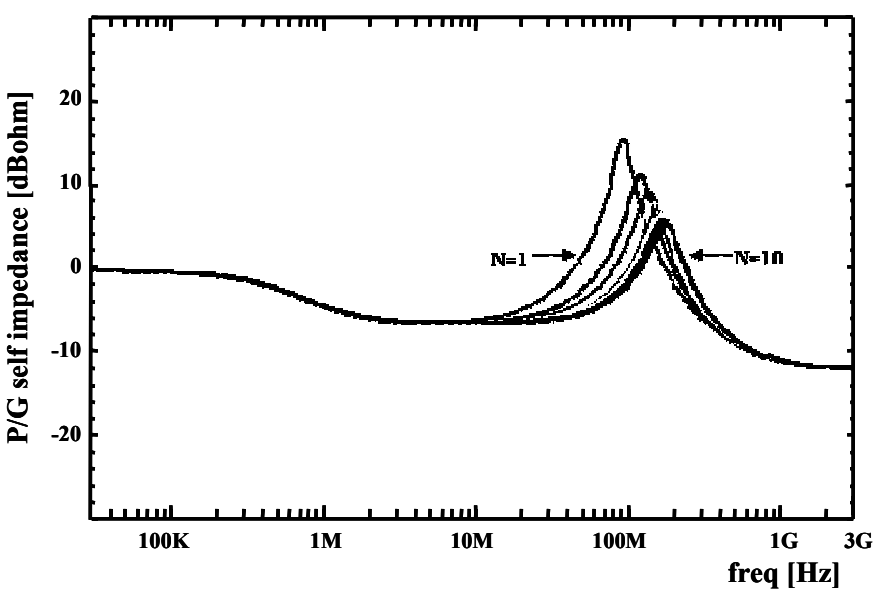

Fig. 8 The effect of the number of bond-wires on power/ground impedance

\section{c. Package Decoupling Capacitor Effect}

The other variable determining the impedance curve before the parallel resonance frequency is the decoupling capacitor of package. Fig. 9 shows the effect of the decoupling capacitor of package on the impedance curve. If the capacitance is too small $(\mathrm{C}$ p pkfdecap $=0.1 \mathrm{nF}, 1.0 \mathrm{nF})$, two resonance peaks give rise to the mid-frequency region and the impedance goes up to around 500mohms. The resonance peak at lower frequency comes from the parallel combination between the capacitance of the decoupling capacitor of package and the equivalent series inductance of the tantalum capacitor which is used for the stability of the VRM. As the capacitance increases, the lower impedance below $1 \mathrm{MHz}$ is obtained, but the parallel resonance peaks at $200 \mathrm{MHz}$ does not changed.

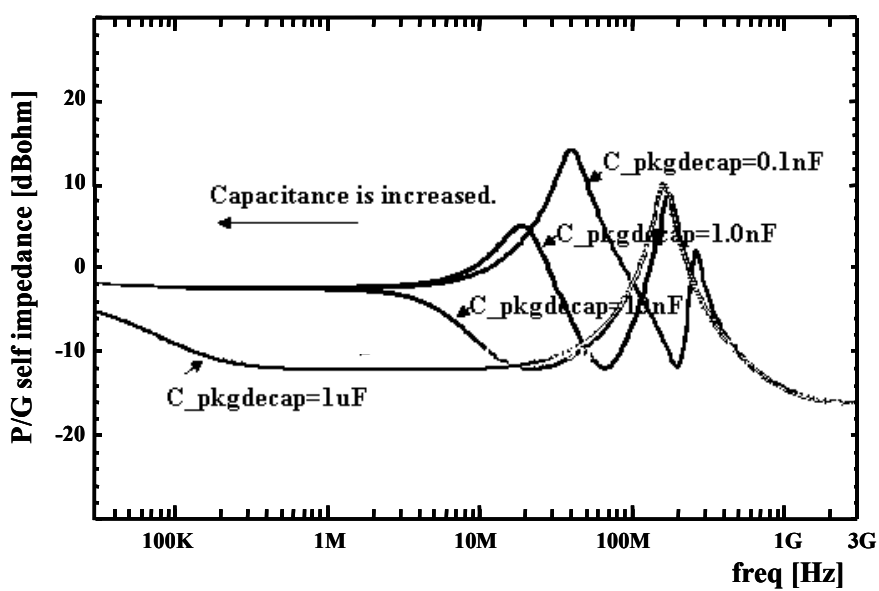

Fig. 9 The effect of the capacitance of the package decoupling capacitor on power/ground impedance

\section{Design Procedure and Analysis for the Resonant Free Power/Ground Network}

Through the analysis, the most effective method to achieve the resonant free power/ground network is to control an equivalent series resistance (ESR) of the on-chip decoupling capacitor. It is known that resistance can lower the Q-factor of the parallel resonance peak. As mentioned before, a conventional on-chip decoupling capacitor is realized with the capacitance between the gate poly and the channel in MOS transistors. Such an on-chip decoupling capacitor has the parasitic resistance that cannot be ignored. The ESR of the on-chip decoupling capacitor can be estimated from the layout dimension of the gate poly and the channel and the number of the on-chip decoupling capacitors. Therefore, the optimized package-chip co-design for the resonant free power/ground network can be achieved through following procedures.

Step I. Set the target impedance according to the allowed noise margin.

Step II. Determine the total ESR of on-chip decoupling capacitors to satisfy the target impedance.

Step III. Choose the total capacitance of the on-chip decoupling capacitors according to the amount of required charge and allowed noise margin. Determine the optimal number and layout dimension of the onchip decoupling capacitors from the total ESR and the total capacitance of the on-chip decoupling capacitor.

Step IV. Make the parasitic inductance in the package and PCB sufficiently low so that the resonance frequency between the capacitance of the on-chip decoupling capacitor and the parasitic inductance can be lower than the frequency where the on-chip decoupling capacitor shows the resistance behavior.

To evaluate the feasibility of the proposed resonant free power/ground network design, the result which is reflected in Fig. 7 and the result from the optimal design are compared. As shown in Fig. 10, the optimal design, which is achieved by 30 on-chip decoupling capacitor in $1600 \mathrm{~mm}^{2}$, makes the impedance at the resonance frequency flat.

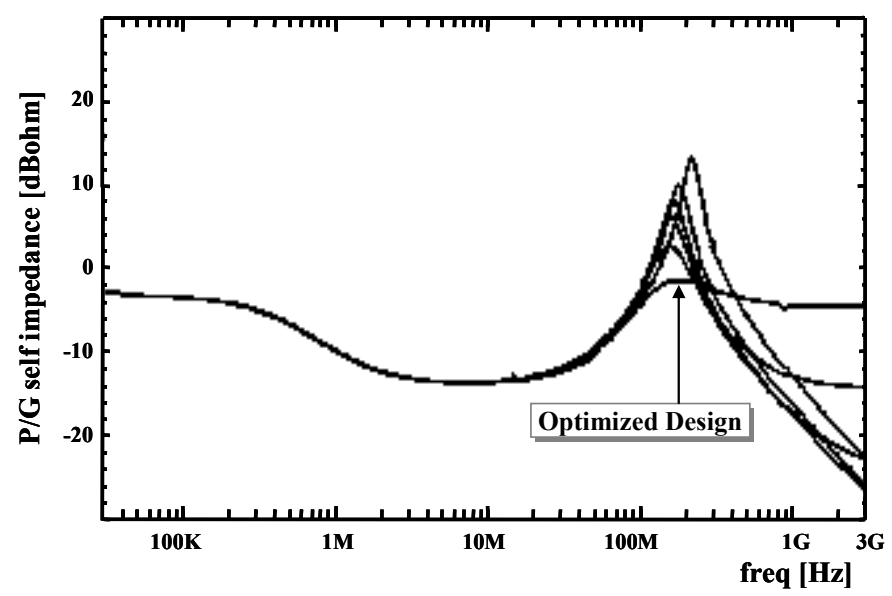

Fig. 10 The optimal design shows the close to flat impedance curve at the resonance frequency.

In the above case, the resistance of on-chip power/ground bus between the noise source and the on-chip decoupling capacitors is lower than $10 \mathrm{mohm}$. However, in reality, the onchip power/ground bus may not be controlled to achieve low impedance. Fig. 11 shows what will happen with the high resistive on-chip power/ground network. The on-chip 
power/ground bus (1) refers to the bus between the noise source and the on-chip decoupling capacitors while the onchip power/ground bus (2) refers to the bus between the onchip decoupling capacitors and the wire-bonding pads. When the impedance curves after the parallel resonance show the resistance dominated behavior, the impedance value is determined by the resistivity of the on-chip power/ground bus (1) as well as the total ESR of the on-chip decoupling capacitors. Therefore, at the step II, the total ESR should be determined to be of the lower value considering the on-chip power/ground bus (1).

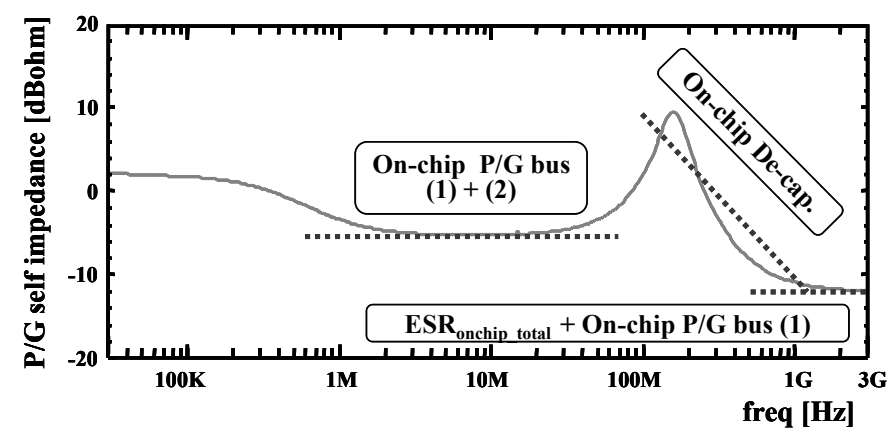

Fig. 11 In the practical case, the on-chip power/ground bus may not be controlled to be low impedance.

\section{Conclusions}

In this paper, it was performed co-modeling and cosimulation about the package and on-chip power/ground network. The model included the parasitic components of the package and the chip. The analysis was executed for the dominant factors for the mid-frequency resonance through the several simulations. From the co-modeling and co-simulation, it has been attested that the most effective way to achieve the resonant free impedance curve is to control the total ESR of the on-chip decoupling capacitor.

\section{References}

1. Larry D. Smith, Raymond E. Anderson, Douglas W. Forehand, Thomas J. Pelc, and Tanmoy Roy, "Power distribution system design methodology and capacitor selection for modern CMOS technology," IEEE Trans on $A P$, Vol. 22, No. 3 (1999), pp. 284-291.

2. Radu, S. and Hockanson, D., "An investigation of PCB radiated emissions from simultaneous switching noise," IEEE International Symposium on Electromagnetic Compatibility, Aug. 1999, Volume: 2 (1999), pp. 893-898

3. Alex Waizman and Chee-Yee Chung, "Resonant Free Power Network Design UsingExtended Adaptive Voltage Positioning (EAVP) Methodology," "IEEE Trans on AP, Vol. 24, No. 3 (2001), pp. 236-244.

4. Jun So Pak, Jingook Kim, Jung-Gun Byun, Heejae Lee, and Joungho Kim, "Coupling of Through-Hole Signal Via to $\mathrm{P} / \mathrm{G}$ Resonance and excitation of edge radiation in Multi-layer PCB," IEEE International Symposium on Electromagnetic Compatibility, Aug. 2003, Volume: 1 (1999), pp. 18-22

5. Gerard Villur. Eduard Alarcdn, Francesc Guinjoan and Alberlo Poveda, "Optimized design of MOS capacitors in standard CMOS technology and evaluation of their Equivalent Series Resistance for power applications," Proc of the 2003 International Symposium on Circuits and Systems, May. 2003, Volume: 3, pp. III-451 - III-454 